Ciencias económicas y empresariales

Artículo de revisión

\title{
Importancia de la enseñanza de la metodología de la investigación científica en las ciencias administrativas
}

\section{Importance of teaching the methodology of scientific research in administrative sciences}

\section{Importância do ensino da metodologia da pesquisa científica em ciências administrativas}

Guadalupe Maria Leonor Toala-Toala I

gtoala2000@hotmail.com

Amado A. Mendoza Briones ${ }^{\text {II }}$

antoniomendoza1971@yahoo.com

Luís M. Moreira-Moreira ${ }^{\mathrm{III}}$

mmoreira.moreira3@gmail.com

Recibido: 10 de enero de 2019 *Aceptado: 10 de marzo de 2019 * Publicado: 05 de abril de 2019

\footnotetext{
${ }^{\text {I }}$ Magíster en Gerencia de Proyectos Educativos y Sociales, Diploma Superior en Educación Universitaria por Competencias, Doctor en Ciencias Administrativas, Licenciado en Ciencias de la Educación Especialidad Físico Matemáticas, Profesor de Segunda Enseñanza Especialidad Físico Matemáticas, Docente Universidad Laica “Eloy Alfaro” de Manabí, Manta, Ecuador.

${ }^{\text {II }}$ Magíster en Gerencia de Proyectos Educativos y Sociales, Diploma Superior en Educación Universitaria por Competencias, Doctora en Ciencias Administrativas, Especialista en Diseño Curricular por Competencia, Ingeniera Comercial, Docente Universidad Laica "Eloy Alfaro" de Manabí, Manta, Ecuador.

III Doctor en Ciencias Administrativas, Magíster en Gerencia de Proyectos Educativos y Sociales, Ingeniero Comercial, Docente Universidad Laica "Eloy Alfaro" de Manabí, Manta, Ecuador.
} 


\title{
Resumen
}

En la presente investigación se razona sobre la determinación de la importancia de la enseñanza de la "Metodología de la Investigación" en la formación de profesionales en la Administración de Negocios, donde se precisa una reflexión acerca de las generalidades de investigación, normatividad para la presentación de trabajos e informes, y la elaboración, sustentación de proyectos y artículos de investigación como principios fundamentales de la proyección en esencia y existencia de la investigación en esta carrera. El administrador como investigador, se forma con acciones de actualización constante de conocimientos orientados al pleno dominio de conocimientos técnicos sobre alguna especialidad en su campo, donde sea capaz de explicar el comportamiento de fenómenos administrativos con base científica y desarrollar propuestas de mejora creativas y adquirir la habilidad para influir en la transmisión, transformación e innovación de nuevos conocimientos. En el mundo actual de globalización y competitividad, las universidades reivindican con mayor fuerza su importancia estratégica para el desarrollo de las sociedades por cuanto tiene la función social de formar un nuevo tipo de profesionales que respondan a las nuevas necesidades del desarrollo; y, lo que es más importante aún, la universidad tiene la misión social de generar Ciencia, Tecnología e Innovación a través de la Investigación Científica, que es un estudio que se enfrenta con el desarrollo de la carrera y también con el desarrollo del trabajo profesional. Se utilizó la investigación bibliográfica-documental y de las conclusiones obtenidas se deduce la importancia de la investigación formativa en las Ciencias Administrativas.

Palabras clave: Metodología de la investigación; investigación científica; tipos de investigación, sociedad del conocimiento; calidad de la investigación.

\begin{abstract}
In the present investigation it is reasoned on the determination of the importance of the teaching of the "Methodology of the Investigation" in the formation of professionals in the Administration of Businesses, where a reflection is needed about the generalities of investigation, normativity for the presentation of works and reports, and the development, support of projects and research articles as
\end{abstract}


fundamental principles of the projection in essence and existence of research in this career. The administrator as a researcher, is formed with actions of constant updating of knowledge oriented to the full mastery of technical knowledge about some specialty in his field, where he is able to explain the behavior of scientific phenomena with scientific basis and develop proposals for creative improvement and acquire the ability to influence the transmission, transformation and innovation of new knowledge. In the current world of globalization and competitiveness, universities claim more strongly their strategic importance for the development of societies because it has the social function of forming a new type of professionals that respond to the new needs of development; and, what is more important, the university has the social mission of generating Science, Technology and Innovation through Scientific Research, which is a study that faces the development of the career and also with the development of professional work . Bibliographic-documentary research was used and the conclusions obtained show the importance of formative research in the Administrative Sciences.

Keywords: Research methodology; scientific research; types of research; knowledge society; quality of research.

\section{Resumo}

Em esta razão, a pesquisa sobre a determinação da importância de ensinar a "Metodologia da Pesquisa" na formação de profissionais em Administração de Empresas, onde uma reflexão sobre as generalidades da pesquisa, é necessário normas para submissão de trabalhos e relatórios, e o desenvolvimento, apoio de projetos e artigos de pesquisa como princípios fundamentais da projeção em essência e existência de pesquisas nesta carreira. $\mathrm{O}$ administrador e pesquisador, é formado com ações constantemente atualizado que visam o controle total do conhecimento técnico de uma especialidade em seu campo, onde ele é capaz de explicar o comportamento de fenômenos administrativos com base científica e desenvolver propostas de melhoria criativa e adquirir conhecimentos a capacidade de influenciar a transmissão, transformação e inovação de novos conhecimentos. No atual mundo da globalização e da competitividade, as universidades reivindicam mais fortemente sua importância estratégica para o desenvolvimento das sociedades, pois tem a função social de formar um novo tipo de profissionais que respondem às novas necessidades de 
desenvolvimento; e, o que é mais importante, a universidade tem a missão social de gerar Ciência, Tecnologia e Inovação através da Pesquisa Científica, que é um estudo que encara o desenvolvimento da carreira e também com o desenvolvimento do trabalho profissional. . Pesquisa bibliográficodocumental foi utilizada e as conclusões obtidas mostram a importância da pesquisa formativa nas Ciências Administrativas.

Palavras-chave: Metodologia de pesquisa; Investigação científica; tipos de pesquisa, sociedade do conhecimento; qualidade da investigação.

\section{Introducción}

La investigación es considerada como un proceso riguroso, cuidadoso y sistematizado en el que se busca resolver problemas, bien sea de vacío de conocimiento (investigación científica), pero en ambos casos es un proceso organizado que garantiza la producción de conocimiento o de alternativas de solución viables. En este artículo es necesario aclarar que se razona sobre la determinación de la importancia de la enseñanza de la "Metodología de la Investigación" en la formación de profesionales en la Administración de Negocios, donde se precisa una reflexión acerca de las generalidades de la investigación, su normatividad para la presentación de trabajos e informes, y la elaboración, sustentación de proyectos y artículos de investigación como principios fundamentales de la proyección en esencia y existencia de la investigación en esta carrera.

El administrador como investigador, se forma con acciones de actualización constante de conocimientos orientados al pleno dominio de conocimientos técnicos sobre alguna especialidad en su campo, donde sea capaz de explicar el comportamiento de fenómenos administrativos y de gerencia de las organizaciones, con base científica y desarrollar propuestas de mejora creativas y adquirir la habilidad para influir en la transmisión, transformación e innovación de nuevos conocimientos.

En el mundo actual de globalización y competitividad, las universidades reivindican con mayor fuerza su importancia estratégica para el desarrollo de las sociedades por cuanto tiene la función social de formar un nuevo tipo de profesionales que respondan a las nuevas necesidades del desarrollo; y, lo que es más importante aún, la universidad tiene la misión social de generar Ciencia, Tecnología e 
Innovación a través de la Investigación Científica, que es un estudio que se enfrenta con el desarrollo de la carrera y también con el desarrollo del trabajo profesional. Se utilizó la investigación bibliográfica-documental y de las conclusiones obtenidas se deduce la importancia de la investigación formativa en las Ciencias Administrativas.

En ese sentido, se asume que la investigación científica es definida como "... un proceso metódico y sistemático dirigido a la solución de problemas o preguntas científicas, mediante la producción de nuevos conocimientos, los cuales constituyen la solución o respuesta a las interrogantes que se hayan generado en un ámbito de conocimiento de las ciencias gerenciales y de la administración en general.

A partir de esto es posible hacer una reflexión acerca del enfoque que de allí se desprende: Si se trata la investigación científica, es posible desarrollar diversas posturas epistémicas, desde las cuales se otorgue atención y solución a los problemas administrativos propios de procesos de gerencia y de dirección de procesos organizacionales, desde los resultados que se obtengan con cierto grado de generalización.

\section{Materiales y Métodos}

\section{La Metodología de la Investigación como asignatura}

La palabra Metodología posee varias acepciones, en primer término, es una palabra compuesta por método (vía o camino para llegar a una meta) y por logos que significa estudio o tratado. Por lo tanto, la Metodología se constituye como el estudio de los métodos empleados para alcanzar un fin. Según Asti Vera (1968), el término Metodología posee dos significados, "una referida al campo de la pedagogía, concretamente relacionada con el estudio de los métodos de enseñanza, y la otra relativa al estudio analítico y crítico de los métodos de investigación y de prueba" (p. 16). Por supuesto, en este artículo se consideró la segunda acepción, es decir, la correspondiente al estudio y análisis de los diferentes métodos de investigación.

Por su parte, para García Avilés (1996), la Metodología de la Investigación es “...la reflexión crítica encargada de estudiar el surgimiento, desarrollo y validez de los métodos empleados en la ciencia..." (p.

60 
65). Resulta claro, que para este autor la Metodología "opera a nivel teórico”, a diferencia de los cursos de Técnicas de Investigación que operan a nivel práctico. Mientras que según Sabino (2000) la Metodología consiste en "...el análisis de los diversos procedimientos concretos que se emplean en las investigaciones...” y coincide con García Avilés en que se deben reservar los términos técnicas y procedimientos para referirse “...a los aspectos más específicos y concretos del método que se usan en cada investigación" (p. 35).

No obstante a ello, la realidad demuestra que los cursos de Metodología de la Investigación impartidos a nivel superior, están centrados más en las técnicas y procedimientos que en la discusión sobre los métodos, entendidos como el modo de obtener un conocimiento científico. De hecho, otros autores como Hernández Sampieri, Fernández y Baptista (2010), así como Arias (2011), se inclinan hacia el enfoque operativo de la Metodología. Este último autor expresa que "la Metodología de la Investigación se ocupa del estudio de los métodos, técnicas e instrumentos que se emplean en el proceso de investigación.” (P. 56) También, esta disciplina analiza los diferentes métodos, técnicas y procedimientos e indica su pertinencia y limitaciones a la hora de ponerlas en práctica.

En este orden de ideas, se asume la Metodología en el mismo sentido adoptado en la Educación Universitaria. Es decir, se entiende como una asignatura que abarca, no sólo la discusión teórica sobre el método, sino también el aspecto operativo referido a las técnicas, procedimientos e instrumentos que se emplean en una investigación. Es importante aclarar que igualmente válida es la acepción del término Metodología como el conjunto de métodos que se emplean en una investigación científica (RAE, 2016).

\section{Importancia de la enseñanza de la Metodología en las Carreras de Administración}

El cambio que sufre un estudiante de la Educación Media al nivel superior universitario supone que el mismo, deja de ser un aceptante de ciencias, para emprender una educación que le permitirá ser crítico y generador de conocimientos. Por lo tanto, la producción de conocimientos o investigación es una tarea inherente a la universidad. Más aún, la mayoría de las normativas internacionales coinciden en señalar la investigación como una función de tanta importancia como la asignada a la docencia. No existe docencia de calidad sin investigación.

\section{1}


La investigación es la representación cognitiva mediante la cual el ser humano puede comprender, analizar, aprender nuevo conocimiento y es por eso que termina resultando importante y central en la vida humana. La investigación puede darse de muchas maneras, algunas más formales y más conscientes que otras, pero es parte vital de nuestra vida como seres en constante aprendizaje y conocimiento. Actualmente, el ejercicio de las funciones universitarias es el resultado de numerosas experiencias y de ensayos, cuya intención no es otra que la de hacer más eficientes las labores de docencia e investigación para obtener un mayor rendimiento en cuanto al aprendizaje se refiere y una mayor productividad en las actividades de investigación.

Entonces la importancia de la Metodología de la Investigación para la formación de los futuros profesionales, quienes, se encuentran en la necesidad de obtener y generar conocimientos relacionados con su especialidad. Por ejemplo, el abogado investigará en el campo jurídico, el médico aplicará técnicas de investigación en el campo de la salud, el educador empleará la Metodología de la Investigación en el aula de clases y el Administrador en el área de la gerencia y la administración, porque ésta permite con una debida fundamentación la reflexión sobre la necesidad de cambios que favorezcan el desarrollo, la productividad en forma eficiente, eficaz y efectiva, dándole primacía al capital humano. Desde esta perspectiva, la investigación está obligada a realizar continuamente revisiones de lo existente. En síntesis, la Metodología, como conjunto de procedimientos y técnicas concretas de indagación, puede aplicarse en cualquier disciplina o área del conocimiento científico.

En ese tenor, según Trujillo, Zambrano y Vargas (2004), la importancia de la Metodología de la Investigación reside en que:

- Permite el análisis reflexivo y crítico de los conceptos teóricos a desarrollar en una investigación.

- Implica la utilización de pasos y procedimientos para resolver problemas cuyas soluciones se hallan por la aplicación del método científico.

- Desarrolla la capacidad de crítica y argumentación a la hora de tomar decisiones frente a los procesos a seguir. 
- Es una herramienta fundamental para el desarrollo de las actividades académicas y científicas.

- Establece el rumbo correcto de una investigación asegurando un trabajo eficaz y eficiente frente a logro de resultados.

Además, la Metodología de la Investigación:

- Presenta y explica las diferentes técnicas para recolectar datos.

- Indica cómo elaborar los instrumentos.

- Señala el porqué de los procedimientos y advierte sobre las consecuencias de obviarlos.

- Suministra información útil para la toma de decisiones.

- Permite la verificación o refutación de juicios emitidos por otras personas.

- Proporciona una base de conocimientos o marco de referencia necesario para fijar posiciones en la vida.

Aunado a lo anteriormente expuesto, el campo de la Administración, se conforma en un mercado competitivo actualmente cada vez más exigente, de manera global busca permanentemente un feedback, es decir persigue resultados científicos que sólo se encuentran en la investigación estructurada, para poder pasar por un tamiz de novedades que hoy permanecen en la praxis gerencial y otras que se consideran obsoletas, así como aquellas que deben innovarse para responder desde el presente a los tiempos por venir, en este sentido, se concilia lo de ayer con lo de hoy y el mañana, organizando todo a unos objetivos enmarcados en la estrategia corporativa.

\section{Relación de la Metodología de la Investigación con otras asignaturas básicas}

La Metodología de la Investigación, se relaciona con todas las áreas del conocimiento en la Educación Superior. Cada materia constituye un objeto o tema de investigación. De esta manera, el estudiante, al cursar cualquier asignatura y al profundizar en un tópico específico, puede plantearse interrogantes o problemas que lo conducirán a la obtención de nuevos conocimientos. Tanto la investigación de campo como la investigación experimental, requieren para su divulgación, la elaboración y presentación de

63 Vol. 5, núm. 2, abril 2019, pp.56-70

Guadalupe Maria Leonor Toala Toala, Amado A. Mendoza Briones, Luís M. Moreira Moreira 
informes escritos. Concretamente, el alumno, al iniciar una indagación, se ejercita en actividades intelectuales básicas como la lectura y la escritura.

En correspondencia con ello, Arturo Uslar Pietri, acertado docto y escritor venezolano, en su obra "Educar para Venezuela" (1981), formuló conceptos y criterios sobre la escuela venezolana, de la cual expresó que era "muda", debido a que no enseñaba a leer ni a escribir. Sin embargo, en la institución educativa, especialmente en la universidad constantemente se exigen informes y diversos tipos de trabajos investigativos. La evaluación del estudiante gira, esencialmente, en torno a la comunicación escrita; por ejemplo, es evidente la relación entre las asignaturas Metodología de la Investigación y Lenguaje y Comunicación.

Igualmente, cuando en una investigación se aplica la técnica de la encuesta, los datos obtenidos son analizados mediante la estadística. He aquí otra relación: Metodología y Estadística. En este sentido, para poder lograr la generalización de los resultados obtenidos en una investigación, se utiliza la inferencia estadística, es decir, técnicas cuantitativas que permiten extrapolar los resultados arrojados en un pequeño grupo de individuos o "muestra", a una población mucho más grande de la cual se extrajo dicha muestra (Salama, 2002).

En resumen, cuando el estudiante aplica la Metodología de la Investigación, simultáneamente pone en práctica otras asignaturas o áreas básicas del conocimiento, por cuanto éstas no se manifiestan de forma aislada, sino que se relacionan, se integran y complementan. De allí que sea pertinente la asunción del procesos de indagación científica desde un enfoque inter y transdisciplinario.

\section{Necesidad mundial de la Investigación Científica en Administración}

En el 2016, el mundo celebró el centenario de la Administración. Fue en 1916 que se publicó la trascendental obra del francés Henry Fayol titulada Administración Industrial y General en la que con una precisión sorprendente se expone, por primera vez en la historia de la humanidad, los fundamentos doctrinarios de la Administración. Han transcurrido 102 años de la Administración universal, sin embargo, en lo esencial, principalmente en su aspecto doctrinario, sigue siendo la misma Administración concebida por sus propulsores clásicos y neoclásicos. Es evidente, por ejemplo, la

64 Vol. 5, núm. 2, abril 2019, pp.56-70 Guadalupe Maria Leonor Toala Toala, Amado A. Mendoza Briones, Luís M. Moreira Moreira 
ortodoxia (y hasta su dogmatismo) de la Administración por cuanto sus originales fundamentos teóricos en lo fundamental no han cambiado y a pesar de su gran literatura, en lo esencial sigue siendo los mismo como lo era hace un siglo.

Por otra parte, en la Administración, -como teoría y como praxis profesional, que como actividad humana es tan antigua como la historia del hombre- aún prevalece una concepción circunscrita solamente a los estrechos marcos de las empresas (y, eventualmente, de las instituciones). Por tal razón, resulta común hablar sólo de la administración de empresas (microadministración) y, de manera circunstancial, de la administración de hospitales, de universidades, de municipalidades, del Estado, del ejército, de las iglesias, etc.; sin percibir que la Administración trasciende más allá de las ceñidas fronteras empresariales e institucionales y se engarza al proceso mismo de desarrollo de las sociedades (macroadministración) y por lo tanto, la Administración adquiere su dimensión de categoría eminentemente política, filosófica e ideológica.

Es en este último contexto que adquiere credibilidad la famosa declaración de Drucker: "Los países subdesarrollados no son tales, son más bien, países subadministrados", o de Deming: "Cualquier país con una buena Administración no tiene por qué ser pobre". Esta bi-dimensionalidad de la Administración conlleva -aunque por ahora cueste admitirlo-, a establecer que la Política, la Economía, el Derecho, la Sociología, la Psicología, etc. son, en su estricta interpretación, categorías inmersas y subordinadas a la Administración.

Es evidente que tales cambios comienzan a impactar a la educación y la investigación en administración en América Latina, cuya evaluación ha sido críticamente negativa por quienes han estudiado el estado de la investigación en administración en la región desde mediados de la década de los ochenta y comienzos de los noventa y que coincidieron en señalar la escasez investigativa y las limitaciones académicas de esta área (Rodríguez et ál,, 1992).

En importante precisar, por otra parte, que pese al primer siglo de la Administración, se sigue subestimando al factor humano como antaño. La gente sigue siendo aún -afrenta aparte- considerada y tratada como "recursos" -incluso los mismos administradores de hoy llaman, despectivamente, 
"recursos humanos" como si las personas fueran máquinas o cosas. El hombre (la persona humana) sigue siendo visto, al igual que hace un siglo, como un medio antes que un fin, desnaturalizando su carácter eminentemente humano de la Administración. No se trata de buscar tecnologías, calidad, productividad, competitividad, mercados; éstos son, más bien, resultados de lo que se haga exactamente lo contrario: buscar prioritariamente calidad y respeto a la gente.

Desde este marco, podemos seguir mencionado un conjunto de limitaciones estructurales de la Administración que evidencian una suerte de estancamiento de su avance científico mundial, máxime en estas últimas tres décadas; empero, todo esto tiene un denominador o causa común: la inexistencia de la Investigación Científica en Administración. No son los Taylor, los Fayol, los McCregor, los Kast, los Mintzberg, los Senge, los Porter, los Hofstede, los Drucker; los que necesariamente abundan en nuestros tiempos; son, más bien, los efímeros best sellers en Administración quienes, sin embargo, no logran remover sus bases doctrinarias. Todo esto demanda renovados esfuerzos en la Investigación Científica en las Ciencias de la Administración capaz de producir nuevos conocimientos, nuevas teorías, tecnologías e innovaciones para contribuir al desarrollo de la humanidad.

\section{Resultados y Discusión}

La asignatura Metodología de la Investigación es fundamental en cualquier programa de formación universitaria, ya que facilita las herramientas para la planificación, ejecución y divulgación de cualquier investigación o proceso dirigido a la obtención de nuevos conocimientos. De igual manera, la Metodología coadyuva a que el estudiante desarrolle una actitud crítica ante la información. Podrá analizar, discernir, evaluar y juzgar juicios emitidos, para luego construir una verdad con la mayor integridad posible, con fundamento en el conocimiento acumulado y con base en los pasos del método científico.

Desde el enfoque funcional, la Metodología ubica al estudiante para la realización de estudiosdiagnósticos, identificación de prioridades y análisis para la toma de decisiones, que contribuyan en la solución de problemas comunitarios, educativos y gerenciales. Por ende se considera que, la Metodología de la Investigación debe ser una asignatura, unidad curricular o área académica obligatoria en todas las 
carreras o programas de formación universitaria y no debe limitarse únicamente a conformar un eje transversal del currículum y es por ellos que los docentes consideran que la Unidad Curricular Metodología de la Investigación en la Carrera de Administración:

Además de impartirse en los primeros semestres debe insertarse como un contenido fundamental en los programas de todas las materias que integran los planes de estudio, de tal modo que el estudiante investigue sobre temas de cualquier asignatura y que se no considere la Metodología sólo como una herramienta necesaria para elaborar el Trabajo Especial de Grado.

Debe enseñarse vinculando la teoría con la práctica. Parafraseando a Carlos Sabino, el estudiante, sólo aprenderá a investigar investigando. Es por eso que los aspectos metodológicos deben relacionarse con todas las áreas y aplicarse en el estudio y análisis de diversos temas. Por ejemplo, si se imparte Metodología a estudiantes de Administración, el profesor deberá enfocar la aplicación de las técnicas de investigación en el abordaje de problemas específicos del campo administrativo.

Para cumplir con lo antes expuesto, el profesor de Metodología de la Investigación, requiere un perfil multidisciplinario, pero principalmente, debe poseer una formación en un área afín con la carrera en la que imparte clases (Arias, 2008). Por ejemplo, un profesor que dicte esta asignatura en la carrera de Informática o Computación, debería poseer los conocimientos básicos de estas disciplinas, así como una formación general en el área tecnológica.

En suma, se considera que las instituciones universitarias deben poner especial atención en la formación, capacitación y actualización de los profesores de Metodología de la Investigación, por cuanto éstos son los responsables directos en la transmisión los conocimientos, habilidades y destrezas que le permitan al estudiante y futuro profesional, la solución de problemas en su campo de acción mediante la aplicación del método científico y la generación de nuevos conocimientos.

La tarea de investigación recae principalmente en los maestros de tiempo completo, pues ellos deben ejercer una labor a veces titánica al combinar la docencia, la investigación y la difusión como ejes fundamentales de su quehacer laboral y universitario. Asimismo, la institución debe seguir intentando

67 Vol. 5, núm. 2, abril 2019, pp.56-70

Guadalupe Maria Leonor Toala Toala, Amado A. Mendoza Briones, Luís M. Moreira Moreira 
establecer mecanismos de apoyo para la investigación y diseñar instrumentos que puedan informar sobre los intereses actuales de los docentes, así como sus necesidades.

De esa manera se podría establecer una proyección de nuevos investigadores, ya que no todos los docentes tienen las mismas necesidades y los mismos intereses. Además, se pueden realizar foros de innovación e investigación que impulsen dicha labor y difundan avances en esta materia; los colegas pueden y deben apoyarse en proyectos de difusión de la ciencia.

Por otro lado, debe apoyarse la productividad, es decir, a quien más produzca se le deben dar estímulos generosos, sin importar que su producción sean artículos, memorias, libros o memorias en extenso. Si bien es verdad, que estos se deben valorar de manera distinta, se puede establecer una base que indique el valor de cada participación; de esa manera el investigador ve que su labor siga siendo dinámica y que su trabajo sea reconocido. Seguramente en el futuro de la facultad estará presente el establecimiento de estrategias actualizadas y pertinentes para procurar el desarrollo de la investigación, ya que siempre se está buscando mejorar la calidad de la universidad. Por último, es consideración de los profesores asumir que la investigación se configura en un indicador importante porque la sociedad generalmente recibe beneficios por esta labor.

\section{Conclusiones}

Los procesos de indagación científica comportan una serie de procedimientos que permiten la generación de conocimientos pertinentes y actualizados, lo cual configura una de las actividades académicas sustantivas de la institución universitaria. Existen diversas definiciones de lo que es el proceso de investigación científica entre los autores de trayectoria como Arias, Hernández, Fernández y Sampieri, entre otros. La convergencia radica en que el proceso busca la solución de problemas en el área de la gerencia y la administración de instituciones, para lo cual la actividad científica destaca por su interdisciplinariedad y pertinencia en áreas del saber específicas y vinculadas a las Ciencias de la Administración.

En ese orden, es consideración de las autoras que la Unidad Curricular Metodología de la Investigación debe trascender a ser una mera asignatura transversal, debe ser una Unidad Curricular que se imparta en

68 
las diversas carreras de formación dirigida por profesores con perfil idóneo no sólo con formación como metodólogos sino además con formación atinente y vinculada con el área de formación de que se trate, es decir los profesores asignados al área de informática con formación en el uso y dominio de las Tic y los profesores asignados al área de la Gerencia y la Administración formados en Contabilidad, en Administración y afines, por ejemplo.

Es por ello que, la Metodología de la Investigación es relevante en los procesos de formación de los estudiantes del área de la Administración, en orden a ofrecer respuestas a las diversas problemáticas de la gerencia, la cultura organizacional y los procesos administrativos correspondientes con la planificación, la organización, la dirección, la ejecución y el control de los recursos materiales y el talento humano que se desempeña en las diversas áreas de la gestión pública y privada que impulsan el desarrollo económico, político y social de un país determinado.

\section{Referencias Bibliográficas}

Arias, F., 2008. Perfil del profesor de Metodología de la Investigación en educación superior. Disponible en: http://www.eumed.net/libros-gratis/2011c/982/

Arias, F., 2011. Metodología de la investigación en las ciencias aplicadas al deporte: un enfoque cuantitativo. Disponible en: http://www.efdeportes.com/efd157/investigacion-en-deporte-enfoquecuantitativo.htm

Asti Vera, A., 1982. Metodología de la investigación. Caracas: Kapelusz.

García Avilés, A., 1996 Introducción a la metodología de la investigación científica. México: Plaza y Valdés.

Hernández Sampieri, R., Fernández, C. y Baptista, P., 2010. Metodología de la investigación $5^{\mathrm{a}}$ ed. México: McGraw-Hill.

Real Academia Española 2016. Diccionario de la lengua española. Disponible en: http://dle.rae.es/

Sabino, C., 2000. El proceso de investigación. Caracas: Panapo.

69 Vol. 5, núm. 2, abril 2019, pp.56-70 Guadalupe Maria Leonor Toala Toala, Amado A. Mendoza Briones, Luís M. Moreira Moreira 
Importancia de la enseñanza de la metodología de la investigación científica en las ciencias administrativas

Salama, D., 2002. Estadística. Metodología y Aplicaciones. Caracas: Ediciones de la Biblioteca de la Universidad Central de Venezuela.

Trujillo, G.; Zambrano, D. y Vargas, A., 2004. Importancia de una metodología de investigación y su relación con el diseño y elaboración de un proyecto investigativo de carácter social. Disponible en: www.manizales.unal.edu.co/modules/unrev_creando/documentos/Metodologia.pdf 